\title{
Analysis of the IMAGE RPI electron density data and CHAMP plasmasphere electron density reconstructions with focus on plasmasphere modelling
}

\author{
T. Gerzen ${ }^{1 *}$, J. Feltens ${ }^{2}$, N. Jakowski1 ${ }^{1}$, I. Galkin ${ }^{3}$, B. Reinisch ${ }^{3,4}$, R. Zandbergen ${ }^{5}$ \\ ${ }^{*}$ German Aerospace Center (DLR), Institute of Communications und Navigation (IKN), \\ Kalkhorstweg 53, D-17235 Neustrelitz, Germany, phone:0049 (0) 3981480 198, fax: 0049 (0) \\ 3981480 123, Tatjana.Gerzen@dlr.de \\ ${ }^{2}$ Telespazio VEGA Deutschland GmbH clo European Space Operations Centre (ESA/ESOC), \\ Robert-Bosch-Straße 2, D-64293 Darmstadt, Germany \\ ${ }^{3}$ University of Massachusetts Lowell, Space Science Laboratory, Lowell, Massachusetts, USA \\ ${ }^{4}$ Lowell Digisonde International, LLC, Lowell, Massachusetts, USA \\ ${ }^{5}$ European Space Operations Centre (ESA/ESOC), Robert-Bosch-Straße 2, D-64293 Darmstadt, \\ Germany
}

\begin{abstract}
The electron density of the topside ionosphere and the plasmasphere contributes essentially to the overall Total Electron Content (TEC) budget affecting Global Navigation Satellite Systems (GNSS) signals. The plasmasphere can cause half or even more of the GNSS range error budget due to ionospheric propagation errors. This paper presents a comparative study of different plasmasphere and topside ionosphere data aiming at establishing an appropriate database for plasmasphere modelling. We analyze electron density profiles along the geomagnetic field lines derived from the Imager for Magnetopause-to-Aurora Global Exploration (IMAGE) satellite / Radio Plasma Imager (RPI) records of remote plasma sounding with radio waves. We compare these RPI profiles with 2D reconstructions of the topside ionosphere and plasmasphere electron density derived from GNSS based TEC measurements onboard the Challenging Minisatellite Payload (CHAMP) satellite. Most of the coincidences between IMAGE profiles and CHAMP reconstructions are detected in the region with L-shell between 2 and 5. In general the CHAMP reconstructed electron densities are below the IMAGE profile densities, with median of the CHAMP minus IMAGE residuals around $-588 \mathrm{~cm}^{-3}$. Additionally, a comparison is made with electron densities derived from passive radio wave RPI measurements onboard the IMAGE satellite. Over the available 2001-2005 period of IMAGE measurements, the considered combined data from the active and passive RPI operations cover the region within a latitude range of $\pm 60^{\circ} \mathrm{N}$, all longitudes, and an L-shell ranging from 1.2 to 15 . In the coincidence regions (mainly $2 \leq \mathrm{L} \leq 4$ ), we check the agreement between available active and passive RPI data. The comparison shows that the measurements are well correlated, with a median residual of $\sim 52 \mathrm{~cm}^{-3}$. The RMS and STD values of the relative residuals are around $22 \%$ and $21 \%$ resp. In summary, the results encourage the application of IMAGE RPI data for plasmasphere and plasmapause modeling.
\end{abstract}

Key words: Plasmasphere, Ionosphere, IMAGE RPI Profiles, CHAMP, Electron Density, Total Electron Content, Plasmapause

\section{Introduction}

The ionosphere is the ionized part of the upper atmosphere extending from about 50 up to 1000 $\mathrm{km}$ height. The plasma is mainly generated by ionizing solar radiation at wave lengths $<130 \mathrm{~nm}$. According to a suggestion by Appleton (1947) the vertical ionization profile may be considered as being composed of different layers (D, E, F1 and F2) depending on major height depending processes and resulting ionization level. The most ionized region of the ionosphere is the F2 layer. The topside ionosphere above the height of the F2 layer peak density changes smoothly into the plasmasphere at a transition height of about $1000 \mathrm{~km}$ height where the atomic oxygen 
and lighter proton densities are equal. The cold plasma of the plasmasphere consisting mainly of light protons, co-rotates with the Earth up the plasmapause height. Here a sharp plasma density gradient separates the dense co-rotating torus-like plasmasphere from the outer magnetosphere (e.g. Davies, 1990; Lemaire et al. 1998).

Only limited data sources are available for studying the plasmasphere, and especially the plasmapause location. Low-power space-borne sounders traversing the whole plasmasphere region, such as the Radio Plasma Imager (RPI) instrument on the Imager for Magnetopause-toAurora Global Exploration (IMAGE) satellite, are rare. During the 2001-2005 period of the IMAGE mission, the RPI instrument measured both close to spacecraft location and remotely sensed electron densities in the Earth's plasmasphere and magnetosphere by (a) observing the natural radio emissions along the orbit, (b) stimulating resonances in the ambient plasma at the spacecraft location, and (c) transmitting and receiving radar signals.

The field-aligned electron density profiles derived by means of radio sounding are of particular interest to our present study. The RPI instrument was the first instrument to observe radar echoes from remote plasma locations at distances up to 7 Earth radii $\left(\mathrm{R}_{\mathrm{E}}\right)$ from the spacecraft where conditions for the specular signal reflection could be met (cf. Reinisch et al., 2001a; Benson et al., 2003; Benson et al., 2013; Section 2). In the RPI plasmagram mode operation, radio pulses with varying frequencies were transmitted and the signal travel times $\tau(f)$ were measured. From these measurements the virtual radar ranges $r^{\prime}(f)$ to the reflecting location are calculated $\left(r^{\prime}=0.5 c \cdot \tau\right.$, where $c$ is the speed of light). A computational procedure inverts the $r^{\prime}(f)$ functions into the electron density functions along the magnetic field line intersecting the spacecraft (cf. Reinisch et al., 2001b; Huang et al., 2004).

In addition to the remote sensing operation, the RPI conducted highly accurate plasma density measurements along the IMAGE satellite orbit, using a passive mode observation (cf. Webb et al., 2007; Denton et. al., 2012; Gerzen et al., 2014), and the relaxation sounding mode measuring characteristic resonance frequencies (cf. Benson et al., 2003). The "sub-pixel" resolution analysis of the relaxation sounding data yields uncertainty of measured plasma frequency as low as 0.1 $\mathrm{kHz}$. Both active and passive techniques determine in-situ plasma density values, though based on slightly different considerations: while the passive method detects the frequency band of the ambient thermal noise bound by the local upper hybrid resonance frequency, the active method triggers the plasma frequency $f_{p e}$ resonance directly.

Dual-frequency Global Navigation Satellite Systems (GNSS) receivers on board of Low Earth Orbiting (LEO) satellites offer additional opportunity of ionospheric sounding, especially of the topside ionosphere and plasmasphere. The Global Positioning System (GPS) is one of these GNSS systems, and its signals were tracked onboard the LEO Challenging Minisatellite Payload (CHAMP), 2000 - 2010 (cf. Reigber et al., 2000). At the German Aerospace Center (DLR), Neustrelitz, Germany, the GPS measurements recorded by CHAMP with its navigation antenna (looking from CHAMP up to the GPS satellites) were used to reconstruct the topside electron density distribution (ionosphere and plasmasphere) from CHAMP up to GPS altitude (cf. Heise et al., 2002). To prove the suitability of these CHAMP topside reconstructions for advanced plasmasphere modelling and for analyzing space weather related changes in the geo-plasma, the accuracy of these data was closely investigated by Gerzen et al. (2014), focusing on the comparison of the CHAMP reconstruction results with the above mentioned electron density data 
derived from passive radio wave observations of the IMAGE RPI instrument for the years 2001 2005.

With the goal of establishing an appropriate database for topside ionosphere, plasmasphere, and plasmapause modelling, we have extended the evaluations performed in Gerzen et al. (2014). In the present paper a comparative study of all above mentioned sources of plasmasphere and topside ionosphere data is conducted. We analyzed the IMAGE electron density profiles and compared them with the CHAMP GPS data derived reconstructions and with in-situ IMAGE electron densities for the years 2001 - 2005. In addition, the availability and data coverage provided by the IMAGE RPI active and passive data are briefly discussed.

The current paper is set up as follows: Details about the IMAGE data are described in Section 2. More details about the CHAMP electron density reconstructions and passive IMAGE data, reference is made to Gerzen et al. (2014). Section 3 explains the data comparison technique. Explicitly addressed are our approach on how to make this different data types comparable, the coincidence criteria and an approach for the coincidence analysis. Section 4 presents the results and is divided into three parts: The first part investigates the comparison of the IMAGE profiles with the CHAMP reconstructions, the second part the comparison of the IMAGE active with IMAGE passive data, and the third part the analysis of both IMAGE active and passive data in terms of availability, data coverage and dependencies. Section 5 summarizes the main conclusions of this study. Finally, Appendix A shows a computation time optimized variant of algorithms used in Section 3.1, which might be helpful for similar applications.

\section{Data}

\subsection{IMAGE RPI data}

The NASA IMAGE (Imager for Magnetopause-to-Aurora Global Exploration) satellite was launched on 25 March 2000. The mission operated successfully until December 2005. Further information about the mission can be found in Burch (2000), Reinisch et al. (2001a; 2009), and Goldstein et al. (2003).

For the work presented in this paper, both in-situ and remote electron density data, obtained from the IMAGE RPI active and passive mode observations were analyzed. Until recently, these secondary data products derived by the RPI team scientists from the original plasmagram and spectrogram data, were not readily available for non-expert use due to significant complexity of the data interpretation and computational expense. In 2015, the Resident Intelligent RPI Archive (cf. Galkin et al., 2008) operated jointly by the University of Massachusetts Lowell (UML) and the NASA Goddard Space Flight Center, began online publishing the plasmagram-derived data and provided the associated data registration documentation to the web portals of the European Near-Earth Space Infrastructure for e-Science (ESPAS) project, cf. Belehaki et al. $(2013,2014)$ and the NASA Virtual Wave Observatory (VWO) at the GSFC.

\subsubsection{Remote sensing of electron density profiles}

Provided by the active sounding operation of the RPI, each profile comprises a list of electron density values along a 3D curve of the geomagnetic field line, given at discrete anchor points and referred to the Solar Magnetic (SM) coordinate system. All density values of the profile are 
tagged with a single reference epoch, even though the exposure time of the plasmagram measurement is in the order of 1-2 minutes. The reference epoch refers to the start of the exposure time interval. In general the profile extends from spacecraft location into both hemispheres along the closed field line within the plasmasphere, but occasionally only one profile segment is available corresponding to signals only in the local hemisphere in the direction of increasing plasma density. For consistency of the analyses, the two hemispheric sections of the profile are treated in the present paper independently. A representative selection of 1157 IMAGE profiles (each typically consisting of two sections) were considered for this study. These profiles are publicly available via the BinBrowser (http://ulcar.uml.edu/rpi_BinBrowser.html). Please note that the UML team is still in process of making public available more IMAGE profiles. Thus, the presented study reflects most probably not all currently available profiles.

\subsubsection{Spectrogram-derived passive mode in-situ densities}

Signals received by RPI in its passive mode are first presented as a function of frequency and time to form a dynamic spectrogram (Galkin et al., 2004). As described by Webb et al. (2007), a semi-automated fitting technique is then applied to extract electron density values. About 205,000 spectrogram-derived records of plasma and gyro-frequency were compiled into a database available for download at http://image.gsfc.nasa.gov. More details can be found in Webb et al. (2007); Denton et al. (2012); Gerzen et al. (2014) and at http://ulcar.uml.edu/rpi.html.

\subsubsection{Plasmagram-derived in-situ plasma densities}

A suite of characteristic resonance and cut-off frequencies of the ambient plasma at the spacecraft location can be evaluated in the plasmagram images (Benson et al., 2003), including the in-situ electron plasma frequency $f_{p e}$ that translates to the local plasma density $\mathrm{Ne}$.

\subsection{CHAMP reconstructed electron density}

The German minisatellite CHAMP (CHAllenging Minisatellite Payload) (cf. Reigber et al., 2000) was launched on July 15, 2000 and re-entered into the Earth's atmosphere on 19 September 2010. The CHAMP orbit was almost circular and near polar with an inclination of $\sim 87$ degrees and an initial altitude of $454 \mathrm{~km}$ that decreased during mission lifetime. The GPS measurements recorded on board CHAMP, with a sampling rate of $0.1 \mathrm{~Hz}$, have been evaluated at the DLR, Neustrelitz, Germany, to reconstruct the 3D electron density structure in the region above the CHAMP orbit up to the GPS altitude. Calibrated link-related topside TEC data derived for each full CHAMP revolution was assimilated into a background model. A full revolution took 93 minutes. The result of the assimilation process is an improved model output with respect to a background, displaying the 93 minutes picture of the 3D topside ionosphere and plasmasphere (Heise et al., 2002; Gerzen et al., 2014). The approach is working locally, meaning that areas far away from the available CHAMP-GPS links remain completely unaffected and display therefore the pure background model output. The data coverage and thus also the influence of the assimilation procedure reach their maximum inside and near the CHAMP orbit plane. For this reason, in analogy to Gerzen et al. (2014), also the present study restricts to the reconstructed electron density along the mean longitude, $\lambda_{C H}$, of the CHAMP orbit plane during a full CHAMP revolution. The CHAMP reconstructions cover the years 2001-2010 and the region between CHAMP and GPS orbit height. 


\section{Coincidence Criteria and Approach for Coincidence Analysis}

Gerzen et al. (2014) describe the method worked out to compare IMAGE/RPI passive radio wave observations with CHAMP reconstructions. In the present paper this method is adapted to the IMAGE profiles showing the comparison of the IMAGE profiles data with CHAMP reconstructions; individual comparisons are conducted per profile.

The next section describes a way how to make electron densities given in a 2D CHAMP topside reconstruction grid comparable to IMAGE RPI electron density profiles given along geomagnetic field lines. Our aim was to make the coincidence decision, not solely based on the satellite location comparison but to find an approach taking into account the whole profile geometry. For this task, since the IMAGE profiles are (non-planar) geomagnetic field lines aligned, we had to find a way to define a kind of a mean plane for each IMAGE profile.

\subsection{Approach for making IMAGE profiles comparable to CHAMP}

The intention here is to find the orientation of the mean IMAGE profile plane and to apply the coincidence criteria, as defined in Gerzen et al. (2014), to that mean plane. If the coincidence criteria are fulfilled for the mean plane, the comparison with the CHAMP reconstruction data is done for all points on that profile. In this section we describe our method for defining the mean IMAGE profile plane. The following input is required:

- Along an IMAGE profile, the derived electron densities $N e(I M)_{i}$ are given as function of their position vectors $\vec{R}_{I M_{i}}$ (cf. Figure 1 ).

- The complete IMAGE profile (i.e. all electron densities recorded along it) refers to one and the same reference epoch, coinciding with the start of the profile measurement time interval (cf. Section 2.1.1).

- The normal unit vector $\vec{\varepsilon}_{C H}$ of the CHAMP reconstruction plane is known (cf. Gerzen et al., 2014).

Figure 1 presents schematically for an IMAGE profile (in green) the position vectors $\vec{R}_{I M_{i}}$ along this profile as red arrows. Using the above input, in the first step the normal vectors of neighboured position vectors along the profile (blue arrows in Figure 1) are computed by cross products:

$\vec{N}_{i, i+1}=\vec{R}_{I M_{i}} \times \vec{R}_{I M_{i+1}}$

In case of a perfect planar IMAGE profile, all position vectors would lie in that plane and all normal vectors would be perpendicular to it and parallel with respect to each other. However, due to the non-planarity of the profile, the normal vectors deviate to a certain extent from parallelism. Nevertheless, the mean per component of these normal vectors (violet arrow in Figure 1) can be understood as indicator of the mean profile plane. The magnitude of each normal vector $\vec{N}_{i, i+1}$ is determined by the area enclosed by the position vectors $\vec{R}_{I M_{i}}$ and $\vec{R}_{I M_{i+1}}$ from which it was computed. This means, those normal vectors representing a larger area will get a higher weight when computing just the mean of the normal vectors: 
$\overrightarrow{\mathrm{N}}_{0}=\frac{1}{k-1} \cdot \sum_{i=1}^{k-1} \vec{N}_{i, i+1} \quad ; \quad \vec{\eta}_{0}=\frac{\overrightarrow{\mathrm{N}}}{|\overrightarrow{\mathrm{N}}|}$

An unweighted mean can be computed with the normal unit vectors:

$$
\overrightarrow{\mathrm{N}}=\frac{1}{k-1} \cdot \sum_{i=1}^{k-1} \frac{\vec{N}_{i, i+1}}{\left|\vec{N}_{i, i+1}\right|} \quad ; \quad \vec{\eta}=\frac{\overrightarrow{\mathrm{N}}}{|\overrightarrow{\mathrm{N}}|}
$$

This unweighted mean normal unit vector $\vec{\eta}$ (cf. Eq. (3)) is used in our study to define the plane of the mean orientation of the considered IMAGE profile.

In Appendix A an optimized form of Eq. (2) is presented, which can be useful for future applications where large amounts of similar data shall be processed. This optimization reduces the number of cross products to be evaluated by $50 \%$.

\subsection{Coincidence Criteria}

To perform the comparison per profile, the following coincidence criteria (cf. Gerzen et al. 2014) are applied now to a complete IMAGE profile, represented by its mean normal unit vector:

1. The IMAGE profile mean normal unit vector $\vec{\eta}$ (cf. Eq. (3)) and the normal unit vector $\vec{\varepsilon}_{C H}$ of the CHAMP reconstruction plane (cf. Gerzen et al., 2014) enclose an angle of $12^{\circ}$ or less.

2. The IMAGE profile reference epoch lies within the CHAMP reconstruction time period, i.e. the Universal Time of the IMAGE profile reference epoch is between the start and the end of the CHAMP data assimilation period (also given in UT).

Once an IMAGE profile is detected to be coincident with a CHAMP 2D reconstruction file, each observation point along the profile is projected into the plane of the CHAMP 2D reconstruction with the method described in Gerzen et al. (2014) to conduct the analyses described in the next section.

\section{Results}

The systematic validation of the CHAMP electron density reconstructions with the very accurate IMAGE passive in-situ electron density measurements, performed by Gerzen et al. (2014), indicates a good correlation between the IMAGE data and the reconstructions for the L-shell $\leq 3$. In this region, the median of the relative CHAMP-minus-IMAGE residuals is about $-3 \%$ and the RMS is around $46 \%$. At high altitudes around the plasmapause the reconstructions seem to underestimate the electron density values, which can be mainly explained by an underestimation produced by the employed background model. In the $4 \leq L \leq 8$ region, the Median of the relative residuals is around $-169 \%$ and the RMS $\sim 156 \%$. Further details are provided in Gerzen et al. (2014). Within this chapter, the outcomes of Gerzen et al. (2014) are tied to the results of the comparison of the IMAGE profiles versus the CHAMP reconstructions and the IMAGE insitu data, presented in the next two subsections.

\subsection{Comparison of IMAGE profile versus CHAMP reconstructions}


For the comparison, the difference between an electron density interpolated in the CHAMP 2D reconstruction grid at the IMAGE projection point, $\mathrm{Ne}(\mathrm{CH})$, and the corresponding electron density value of the point on the IMAGE profile, $\mathrm{Ne}(\mathrm{IM})$, is calculated for each observation point along the profile according to $d N e=N e(C H)-N e(I M)$.

In addition, relative residuals versus the IMAGE electron density are investigated:

$d N e_{\%}=\frac{N e(C H)-N e(I M)}{N e(I M)} \cdot 100 \%$

For statistical analysis of the CHAMP minus IMAGE electron density residuals, in a pre-step the mean and the Standard Deviation (STD) of the differences were computed, and values outside of the mean $\pm 2.576 \cdot S T D$ bounds were excluded from the analysis. Assuming a Gaussian distribution of the difference values, these margins lead to an exclusion of about $1 \%$ of all values. This was performed in order to avoid a distortion of the statistical values through extreme outliers.

In the following, additionally to the median and RMS of the residuals, also the $90 \%$ quantile of the absolute values $|d N e|$ and $\left|d N e_{\%}\right|$ were computed, by increasingly sorting of the absolute values and calculating the nine tenths bound. In summary, the above described statistical approach is closely aligned to the method described by Gerzen et al. (2014).

Figure 2 presents exemplarily for two days the interpolated CHAMP (magenta dots) profiles and the IMAGE (cyan stars) profiles versus the L-shell. The fix CHAMP longitude $\lambda_{C H}$ is equal to $66.5^{\circ} \mathrm{E}$ for the left - and $136.5^{\circ} \mathrm{E}$ for the right hand panel. The geographic latitude of the IMAGE profile is varying for the left hand panel between 16.4 and $40.5^{\circ} \mathrm{N}$ and for the right hand panel between 32.9 and $51.3^{\circ} \mathrm{N}$. The IMAGE profile longitude is varying for the left hand panel between -77.7 and $-79.4^{\circ} \mathrm{E}$ and for the right hand panel between 137 and $138.6^{\circ} \mathrm{E}$. For both example days the CHAMP electron densities are significantly lower than those of the IMAGE profiles. It is important to remember here, that the IMAGE values are presented in the figure as they appear in the RPI profile, whereas the depicted CHAMP values are deduced from the reconstruction by the projection and interpolation procedure. This is most probably the reason for the non-smoothness of the CHAMP profile shape.

The majority of coincidences fall into the year 2001, further at the end of 2002 and at the beginning of 2004. Figure 3 presents the scatterplot (left hand side) of all coincident IMAGE profiles versus interpolated CHAMP electron densities. As in the comparison with passive IMAGE data (Gerzen et al., 2014), we observe also here that the CHAMP electron densities are predominantly lower than the IMAGE data. The histogram of the $d N e$ values on the right hand side subfigure substantiates this fact, showing also the median, RMS and the $90 \%$ quantile (related to $|\mathrm{dNe}|$ ) values of the CHAMP-IMAGE residuals. The magnitude of the residual statistics is significantly higher than of those between CHAMP and passive IMAGE data, presented in Gerzen et al. (2014). This may result from the differences in the comparison approaches: In Gerzen et al. (2014) local electron densities are tested for coincidence and compared, whereas in the present work the profiles are tested for coincidence and then the 
electron density value of each observation point along the whole IMAGE profile is compared with the corresponding projected and interpolated value in the CHAMP 2D reconstruction plane.

Figure 4 depicts the relative residuals $d N e_{\%}$ versus L-shell on the left hand side and versus geographic latitude of the projected IMAGE observation points on the right hand side. The coincident profile values are mostly in the L-shell range $2 \leq L \leq 5$ and between latitudes of -30 and $50^{\circ} \mathrm{N}$. These two subfigures indicate again that the magnitudes of the CHAMP electron densities are below those of the IMAGE profiles. The $d N e_{\%}$ values range mainly between 70 and $-150 \%$.

Table 1 shows the statistics of the relative residuals for L-values (from top to bottom): $1 \leq L \leq 3$; $3 \leq L \leq 4$ and $4 \leq L \leq 8$. The corresponding mean values (not depicted in the table) arranged again in the same order are around: $-36 \% ;-39 \% ;-21 \%$. Considering the RMS and the $90 \%$ quantile, an increase with growing L-shell is visible, whereas the highest median value is observed for the L-shell range between 3 and 4. For this region, the median is similar to the comparison with passive IMAGE RPI data presented in (Gerzen et al., 2014, Fig. 9). Contrary, the RMS and 90\% quantile are smaller in this study. Furthermore, for $L \leq 3$, the median of the relative IMAGE profile residuals is around $-45 \%$ and hence significantly higher than the median in (Gerzen et al., 2014, Fig. 8, around -3\%). Again, as in the case of Figure 3, this may be at least partly explainable by the differences in the comparison approaches applied here and in Gerzen et al. (2014). In general, we do not observe the same strong correlation between growing L-shell and increasing relative residuals as in the comparison with passive IMAGE RPI data (cf. Gerzen et al., 2014, Figs. 8 and 9).

Reviewing the results presented in Figure 3, Figure 4 and Table 1, we have to keep in mind that the IMAGE data for these comparisons are taken directly from the RPI profiles, whereas the CHAMP values, $\mathrm{Ne}(\mathrm{CH})$, are strongly affected by the projection and interpolation procedure used to calculate them.

\subsection{Comparing IMAGE active and IMAGE passive mode electron densities}

Benson et al. (2004) conducted IMAGE/RPI passive versus active comparisons by means of plasma frequency $f_{p e} \propto N e^{1 / 2}$ comparisons obtained with these two measurement techniques. Their study concluded with the result that the frequency values obtained from both IMAGE techniques, passive and active, agreed within a few per cent (typically 5\%).

In this chapter, comparisons of IMAGE passive versus active data are conducted directly in terms of electron density. For this task the following coincidence criteria are applied:

1. Temporal coincidence between passive and active data: The absolute time difference between the IMAGE passive and IMAGE active data epoch does not exceed 10 minutes.

2. Spatial coincidence between passive and active data: The spatial difference in geographic latitude / geographic longitude / L-shell value between corresponding IMAGE passive and active observation points is less or equal to $5 \% 5^{\circ} / 400 \mathrm{~km}$ respectively.

Figure 5 presents the spatial distribution of the coincident IMAGE passive (black dots) and active (cyan stars) data. Displayed are the L-shell distances (left panel) and the geographic latitude 
(right panel). As stated in Section 2.1, all records of one IMAGE profile refer to the same reference epoch even though their measurement spans 1-2 minutes, with the reference epoch coinciding with the start of the profile measurement time interval. Therefore several IMAGE profile electron density values may satisfy the above selection criteria for comparison against the only one passive IMAGE electron density value (cf. Figure 5). When more than one density value from a profile matches a passive electron density value, we use the inverse distance weighted mean of all coincident profile electron density values.

A total of about 11000 coincident IMAGE passive versus active observation points were detected. By the averaging procedure the number of coincidences for the comparison was reduced to around 1000 covering the years 2001 - 2005. The majority of the coincident observations is located between -50 and $50{ }^{\circ} \mathrm{N}$ geographic latitudes, between $\mathrm{L}=2$ and $\mathrm{L}=4$ and is distributed over all longitudes.

The left hand side of Figure 6 presents the scatter plot of the IMAGE passive versus averaged coincident IMAGE profiles electron density values. The IMAGE passive data are plotted along the horizontal axis and the IMAGE profiles data along the vertical axis. A good correlation between both data types is recognizable, with a slight tendency to the left, meaning that the profiles electron densities are slightly higher than the passive mode densities. The histogram of the residuals on the right hand side subfigure confirms this: The whole residuals distribution is slightly shifted to the negative values as well as the Median value.

Figure 7 shows the distribution of the relative passive-minus-active residuals (calculated according to Eq. (4) and replacing $\mathrm{Ne}(\mathrm{CH})$ by passive mode IMAGE electron density values) versus L-shell on the left hand side and versus geographic latitude of the passive mode measurements on the right hand side. The majority of the relative residuals are ranging between $\pm 30 \%$. No clear correlation is visible between the latitudes of the observation points and the magnitude of the relative residuals, the same holds for L-shell distances. The Median, RMS and STD values of the relative residuals (not depicted on the figure) are: $-5.2 \%, 21.6 \%$ and $20.9 \%$.

\subsection{Spatial analysis of the IMAGE active and passive mode data}

Throughout this subchapter we consider all available (not just the coincident) IMAGE active and passive mode data. The geographic positions of all considered IMAGE (active and passive) observation points are displayed on the left hand side of Figure 8. The right hand side panel depicts the geographic latitudes versus L-shell of the observation points. The positions of the active data are marked cyan, the passive data black. Covered are areas within the latitude range of $\pm 60^{\circ} \mathrm{N}$ and all longitudes. For the region $L \leq 4$, all latitudes between $\pm 60^{\circ} \mathrm{N}$ are present in the data. In the region $4 \leq L \leq 8$, the passive data are available for latitudes between $\pm 40^{\circ} \mathrm{N}$ and the profiles for latitudes between 20 and $60^{\circ} \mathrm{N}$. For L-shell $>10$ almost only IMAGE passive data are present. Additionally the dependency between the IMAGE electron density and L-shell is roughly presented in Figure 9. Please note that the figure shows all available IMAGE data, not only the coincident ones.

With regard to the establishment of a database for an anticipated new plasmasphere model, based on the analyses of Gerzen et al. (2014) and the current study, the data coverage situation looks quite optimistic. However, one has to keep in mind that Figure 8 presents all five years 20012005 together. The data availability for the year 2001 only is shown exemplarily in Figure 10. So 
data gap problems may arise when trying to bin the data for model fitting into certain periods, e.g. of different solar and/or magnetic activity.

\section{Conclusions}

An analysis of plasmasphere and topside ionosphere electron density data from different sources is presented in this paper. The main focus is on the IMAGE RPI electron density profiles given along geomagnetic field lines and their suitability for plasmasphere modelling. These data were compared with CHAMP topside ionosphere and plasmasphere reconstructions and the very precise IMAGE RPI in-situ data. The present study is a follow up work to the evaluations performed in Gerzen et al. (2014), where the CHAMP reconstructions were systematically validated with the IMAGE RPI passive measurements to investigate their potential for analysing the geo-plasma.

The majority of the considered IMAGE profiles are recorded in the years 2001, 2002 and 2004, covering all longitudes and the L-shell distances between 1.6 and 5. The IMAGE RPI profiles and passive data are well correlated, which is indicated by median, RMS and STD values of the corresponding relative residuals of around $-5 \%, 22 \%$ and $21 \%$ resp. The comparison between IMAGE profiles and CHAMP reconstructions shows that the CHAMP reconstructions estimate the electron density systematically below the IMAGE values. The highest relative residuals appear in the region with L-shell values between 3 and 4 and above 5. The majority of the IMAGE profiles versus CHAMP reconstructions coincidences are located in the region with $2 \leq \mathrm{L} \leq 5$, geographic latitude ranging from -40 to $50{ }^{\circ} \mathrm{N}$ and the year 2001 .

Reviewing this results, we have to be aware that the IMAGE profiles data for these comparisons are not manipulated (taken directly as they were estimated from the active RPI measurements), whereas the CHAMP values are strongly affected by the applied projection and interpolation procedure. The relatively high magnitude of the residuals may be further at least partly caused by the applied comparison approach: the IMAGE profiles in whole are tested for coincidence with CHAMP reconstructions and then the electron density value of each observation point along the whole IMAGE profile is compared with the corresponding projected and interpolated value in the CHAMP 2D reconstruction plane.

The following points recapitulate the outcomes derived in the present study and relate them to the results presented in Gerzen et al. (2014):

1. The accurate IMAGE in-situ electron density measurements are well suitable for plasmasphere modelling, but of limited availability (just along the IMAGE orbit).

2. Gerzen et al. (2014), indicate a strong correlation between the IMAGE passive data and the CHAMP reconstructions for the L-shell distances below 3. Hence also the CHAMP reconstructions provide an appropriate database (with significantly more data compared to the IMAGE passive data) for the modelling of this region.

3. In the $3 \leq L \leq 8$ region, the CHAMP reconstructions seem to underestimate the electron density values measured by IMAGE in passive mode, mainly explainable by the employed background model (cf. Gerzen et al., 2014). 
4. The majority of the coincidences between the IMAGE profiles and CHAMP data are detected in the region $2 \leq L \leq 5$ (cf. Figure 4). Further, the magnitudes of the CHAMP electron densities are significantly below those of the IMAGE profiles, especially in the regions $3 \leq L \leq 4$ and $L \geq 5$ (cf. Figure 3 and Figure 4).

5. The majority of the coincidences between the IMAGE active and IMAGE passive data are detected in the region $2 \leq L \leq 4$. Further, a strong correlation between the coincident passive and active IMAGE data is indicated in all available altitudes, also in the region $L \geq 3$. (cf. Figure 5, Figure 6 and 7).

Combining the outcomes summarized in the points 1, 2 and 5 we can conclude that both IMAGE passive and active data are well suited for the plasmasphere modelling. However, the conducted comparison between passive and active IMAGE data is naturally restricted to locations along the satellite orbit.

As stated already in Gerzen et al. (2014), the CHAMP profiles are appropriate for the topside ionosphere/lower plasmasphere modeling up to $L \leq 3$ but not for higher altitudes.

Overlooking the results summarized in the points 1,3 , and 4 and assuming consistency of the IMAGE profiles, it seems to be realistic, that also the IMAGE profiles reflect the electron density distribution of the upper plasmasphere more realistically than the CHAMP profiles. This leads to the conclusion that the IMAGE data (passive and active) appears to be more appropriate for the upper plasmasphere/plasmapause modeling than CHAMP profiles.

Taking into account the spatial and temporal availability of the CHAMP reconstructions, IMAGE active and passive measurements, and the potential of the data for different ionospheric/plasmaspheric regions, the establishment of a database for a new plasmasphere model is quite promising. However, the available data may become insufficient when trying to bin the data for model fitting into certain locations and periods covering different solar and magnetic activity.

\section{Acknowledgments:}

The authors thank A. Kozlov from the University of Massachusetts Lowell for his help with access to the IMAGE RPI profiles. We thank our colleague David Minkwitz from the German Aerospace Center for discussions and the support during the revision process. The authors are thankful to R.E. Denton for providing the IMAGE RPI passive electron density data. The associated work at Dartmouth College (R.E. Denton) was further supported by the US National Science Foundation Grant ATS 1105790. The authors are grateful to all colleagues of the CHAMP team. The authors thank the journal Editor, Jan Laštovicka, and the reviewers for their helpful comments by evaluating our paper.

\section{Appendix A}

The evaluation of Eqs. (2) and (3) requires the computation of a sequence of cross products. The number of cross products to be evaluated can be quite large, up to several hundred per IMAGE profile. To optimize computation, for Eq. (2), the sum of cross products can be re-formulated in terms of vector differences as follows: 
$\overrightarrow{\mathrm{N}}_{0}=\sum_{i=1, \Delta i=2}^{k-2} \vec{R}_{I M_{i+1}} \times\left(\vec{R}_{I M_{i+2}}-\vec{R}_{I M_{i}}\right) ; \quad \vec{\eta}_{0}=\frac{\overrightarrow{\mathrm{N}}}{|\overrightarrow{\mathrm{N}}|}$

(5)

Note that in Eq. (5) the sum is now incremented in steps of $\Delta i=2$, thus the number of cross products to be evaluated is reduced to $50 \%$. Eq. (5) covers exactly all cross products, if the total number $k$ of IMAGE profile position vectors is odd. In the case of an even number of IMAGE profile position vectors, the last cross product of the sum is not covered and has to be evaluated in the normal way and attached to the end of the sum:

$\overrightarrow{\mathrm{N}}_{0}=\left\{\sum_{i=1, \Delta i=2}^{k-2} \vec{R}_{I M_{i+1}} \times\left(\vec{R}_{I M_{i+2}}-\vec{R}_{I M_{i}}\right)\right\}+\left(\vec{R}_{I M_{k-1}} \times \vec{R}_{I M_{k}}\right) ; \quad \vec{\eta}_{0}=\frac{\overrightarrow{\mathrm{N}}}{|\overrightarrow{\mathrm{N}}|}$

For the sum of normal unit vectors, Eq. (3), such an optimization could not be implemented. For the number of IMAGE profiles considered here, this optimization imposed no significant benefits. However it may be of relevance for similar applications where more data needs to be processed.

\section{References}

Appleton, E. V., The ionosphere, 1947. Nobel Lecture, December 12, 1947.

Belehaki, A., M. Hapgood and the ESPAS Team, 2013. ESPAS, the near-Earth space data infrastructure for e-Science: architecture, data model and first release. EGU 2013, Vol. 15, EGU2013-8601, 2013.

Belehaki, A., M. Hapgood, N. Manola, S. Ventouras, S. James, G. Athanasopoulos, A. Lempesis, S. Marziou, J. Watermann, and the ESPAS team, 2014. THE ESPAS E-INFRASTRUCTURE: ACCESS TO DATA FROM NEAR-EARTH SPACE. Proc. of BiDS'14, 12-14 November 2014, online at: http://dx.doi.org/10.2788/1823 (p. 350-353), doi: 10.2788/1823, 2014.

Benson, R. F., Osherovich, V. A., Fainberg, J., and Reinisch, B. W., 2003. Classification of IMAGE/RPI-stimulated plasma resonances for the accurate determination of magnetospheric electron-density and magnetic field values. J. Geophys. Res., 108(A5), 1207, doi:10.1029/2002JA009589, 2003.

Benson R. F., Webb, P. A., Green, J. L., Garcia, L., and Reinisch B. W., 2004. Magnetospheric electron densities inferred from upper-hybrid band emissions. Geophys. Res. Lett., 31, L20803, doi:10.1029/2004GL020847, 2004.

Benson, R. F., Vinas A. F., Osherovich V. A., Fainberg J., Purser C. M., Adrian M. L., Galkin I. A., and Reinisch B. W., 2013. Magnetospheric electron-velocity-distribution function 
information from wave observations. J. Geophys. Res. Space Physics, 118, 5039-5049, doi:10.1002/jgra.50459, 2013.

Burch, 2000. J.L. IMAGE mission overview. Science 291, 619-624, 2000.

Davies, K., 1990. Ionospheric Radio. IEE Electromagnetic Wave Series, 31, Peter Peregrinus Ltd., ISBN 086341186 X.

Denton, R. E., Wang, Y., Webb, P. A., Tengdin, P. M., Goldstein, J., Redfern, J. A., Reinisch, B.W., 2012. Magnetospheric electron density long-term (>1 day) refilling rates inferred from passive radio emissions measured by IMAGE RPI during geomagnetically quiet times. J. Geophys. Res., 117, A03221, doi:10.1029/2011JA017274, 2012.

Galkin, I.A., Reinisch, B. W., Grinstein G., Khmyrov, G., Kozlov, A., Huang, X., and Fung, S. F., 2004. Automated exploration of the radio plasma imager data. J. Geophys. Res., 109(A12), A12210, doi:10.1029/2004JA010439, 2004.

Galkin, I.A., Khmyrov, W., Kozlov, A., Reinisch, B.W., 2008. Intelligent resident archive for RPI level 2 data, in Radio Sounding and Plasma Physics, AIP Conf. Proc., 974, 111-117, 2008.

Gerzen, T., Feltens, J., Jakowski, N., Galkin, I., Denton, R., Reinisch, B. W., and Zandbergen, R., 2014. Validation of plasmasphere electron density reconstructions derived from data on board CHAMP by IMAGE/RPI data. Adv. Space. Res., 55(1), 170 - 183 , doi:10.1016/j.asr.2014.08.005, 2014.

Goldstein, J., Spasojević, M., Reiff, P. H., Sandel, B. R., Forrester, W. T., Gallagher, D. L., Reinisch, B. W., 2003. Identifying the plasmapause in IMAGE EUV data using IMAGE RPI in situ steep density gradients. J. Geophys. Res., 108(A4), 1147, doi: 10.1029/2002JA009475, 2003.

Heise, S., Jakowski, N., Wehrenpfennig, A., Reigber, Ch., Lühr, H., 2002. Sounding of the topside ionosphere/plasmasphere based on GPS measurements from CHAMP: Initial results. Geophys. Res. Lett., 29(14), DOI: 10.1029/2002GL014738, 2002.

Huang, X., Reinisch, B. W., Song, P., Green, J. L., and Gallagher, D. L., 2004. Developing an empirical density model of the plasmasphere using IMAGE/RPI observations. Adv. Space. Res. $33,829-832,2004$.

Lemaire, J. F., Gringauz, K. I., Carpenter, D. L., and Bassolo, V., 1998. The Earth's Plasmasphere, Cambridge University Press, Atmospheric and Space Science Series, 1998.

Reigber, Ch., Lühr, H., Schwintzer, P., 2000. CHAMP mission status and perspectives. Suppl. to EOS, Transactions, AGU, 81(48), F307, 2000.

Reinisch, B.W., Huang, X., Haines, D.M., Galkin, I.A., Green, J.L., Benson, R.F., Fung, S.F., Taylor, W.W.L., Reiff, P.H., Gallagher, D.L., Bougeret, J.L., Manning, R., Carpenter D.L., 
Boardsen S.A., 2001a. First results for the Radio Plasma Imager on IMAGE. Geophys. Res. Lett., 28(6), 1167 - 1170, 2001a.

Reinisch, B. W., Huang, X., Song, P., Sales, G. S., Fung, S. F., Green, J. L., Gallagher, D.L., and Vasyliunas, V. M., 2001b. Plasma Density Distribution Along the Magnetospheric Field: RPI Observations From IMAGE. Geophys. Res. Lett., 28(24), 4521 - 4524, 2001 b.

Reinisch, B. W., Mark, W., Moldwin, B., Denton, R. E., Gallagher, D. L., Matsui, H., Pierrard, V., Tu, J., 2009. Augmented Empirical Models of Plasmaspheric Density and Electric Field using IMAGE and CLUSTER Data. 2009. Space Sci. Rev., 145, 231-261, doi:10.1007/s11214-0089481-6.

Webb, P. A., Benson, R. F., Denton, R. E., Goldstein, J., Garcia, L. N., and Reinisch, B. W., 2007. An Inner-Magnetospheric Electron Density Database Determined from IMAGE/RPI Passive Dynamic Spectra. Eos Trans. AGU, 88(52), Fall Meet. Suppl., Abstract SM12A-04.

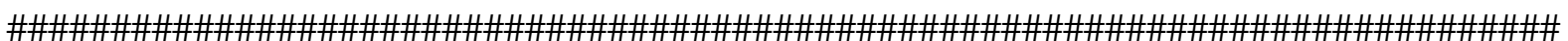

Figure Captions:

Figure 1: Vector geometry to find the mean plane of an IMAGE profile.

Figure 2: Electron density profiles for DOY 148 of 2001 (left) and DOY 036 of 2004 (right). The interpolated CHAMP profile is displayed in magenta and the IMAGE profile in cyan.

Figure 3: Left: Scatter plot of the IMAGE profiles (horizontal) versus CHAMP (vertical) electron density values. Right: The histogram of the dNe values.

Figure 4: $d N e_{\%}$ values versus L-shell (left) and versus geographic latitude (right).

Figure 5: L-shell (left) and geographic latitude (right) of the IMAGE active (cyan stars) and passive (black dots) coincident data.

Figure 6: Left: Scatter plot of the IMAGE passive (horizontal) versus averaged profile (vertical) electron density values. Right: The histogram of the IMAGE in-situ minus active residuals.

Figure 7: Relative IMAGE residuals versus L-shell (left) and versus geographic latitude (right).

Figure 8: Locations of all available IMAGE active (cyan) and passive (black) observation points for years 2001-2005. Left: Geog. longitude versus latitude, Right: Geog. latitude versus L-shell.

Figure 9: IMAGE electron density (passive - black and active - cyan) versus L-shell for years 20012005.

Figure 10: Locations of all available IMAGE active (cyan) and passive (black) observation points for year 2001. Left: Geog. longitude versus latitude. Right: Geog. latitude versus L-shell. 
Table Captions:

Table 1: The statistics of the $d N e_{\%}$ values binned to L-shell ranging between (from top to bottom): 1 and 3; 3 and $4 ; 4$ and 8 . 


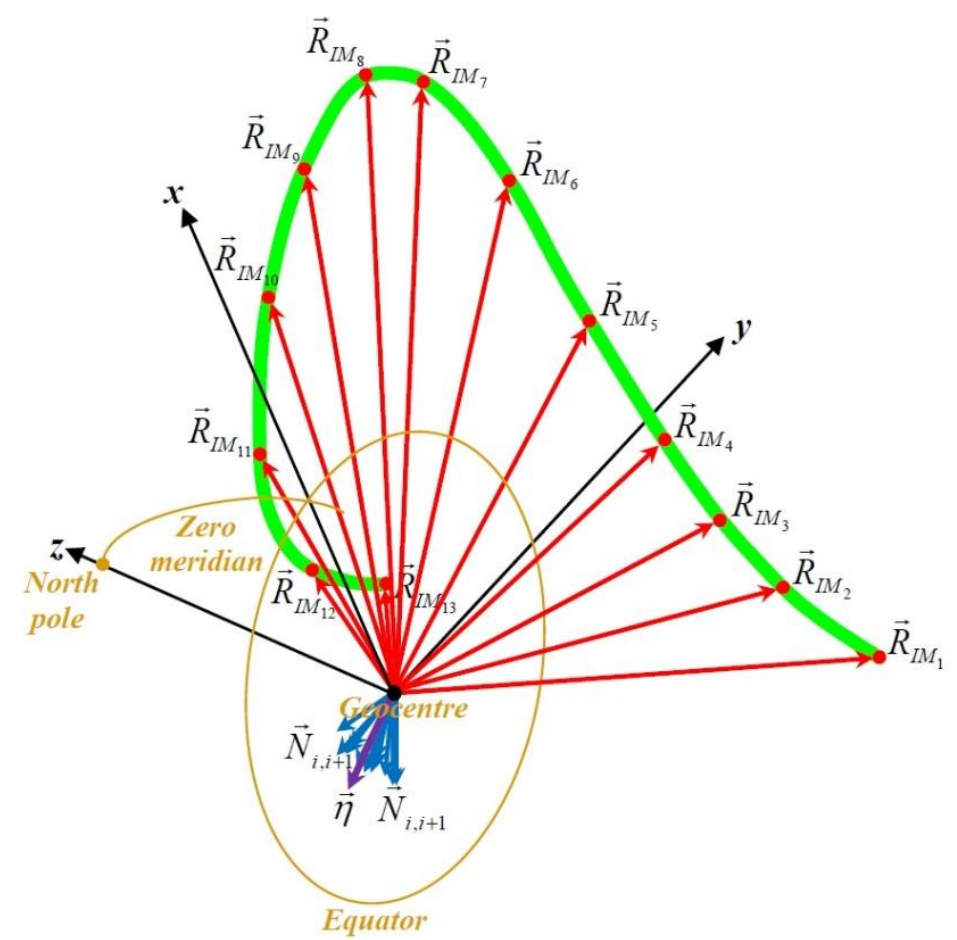

Figure 1: Vector geometry to find the mean plane of an IMAGE profile.
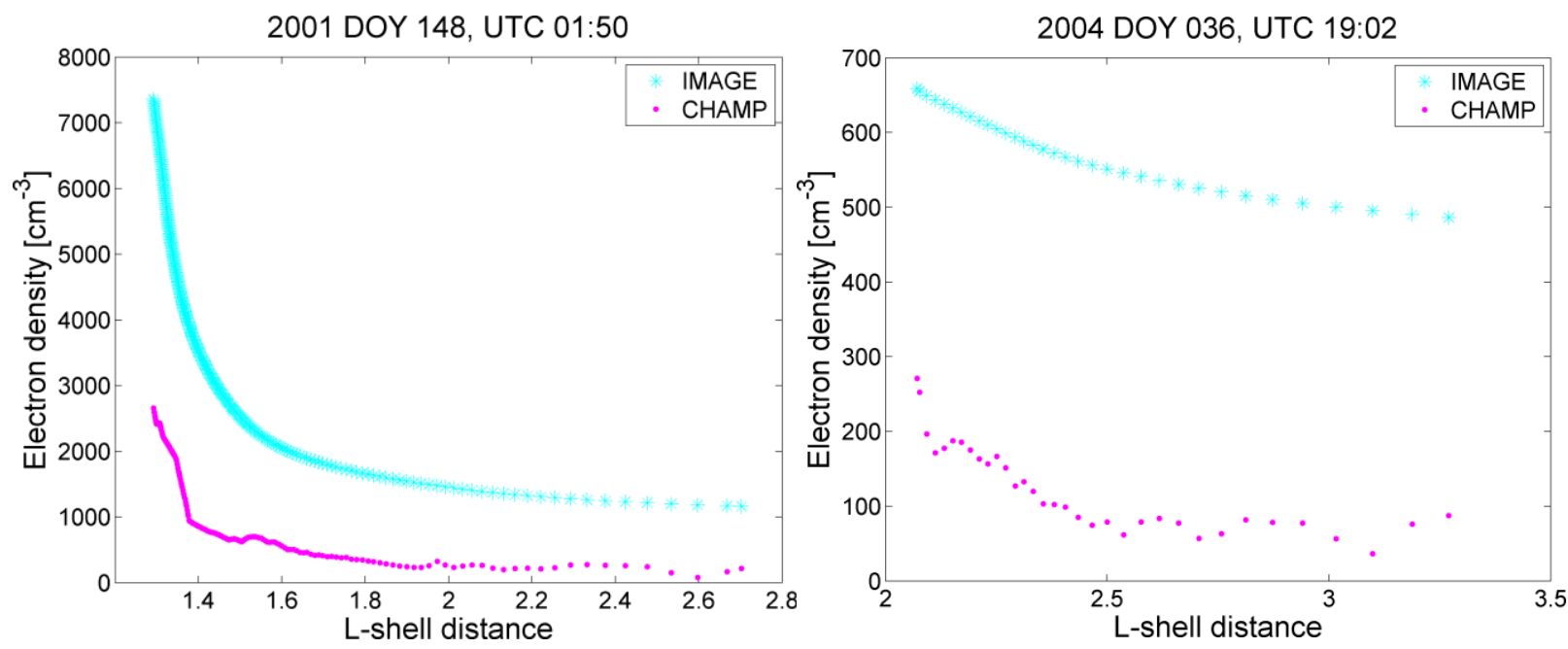

Figure 2: Electron density profiles for DOY 148 of 2001 (left) and DOY 036 of 2004 (right). The interpolated CHAMP profile is displayed in magenta and the IMAGE profile in cyan. 

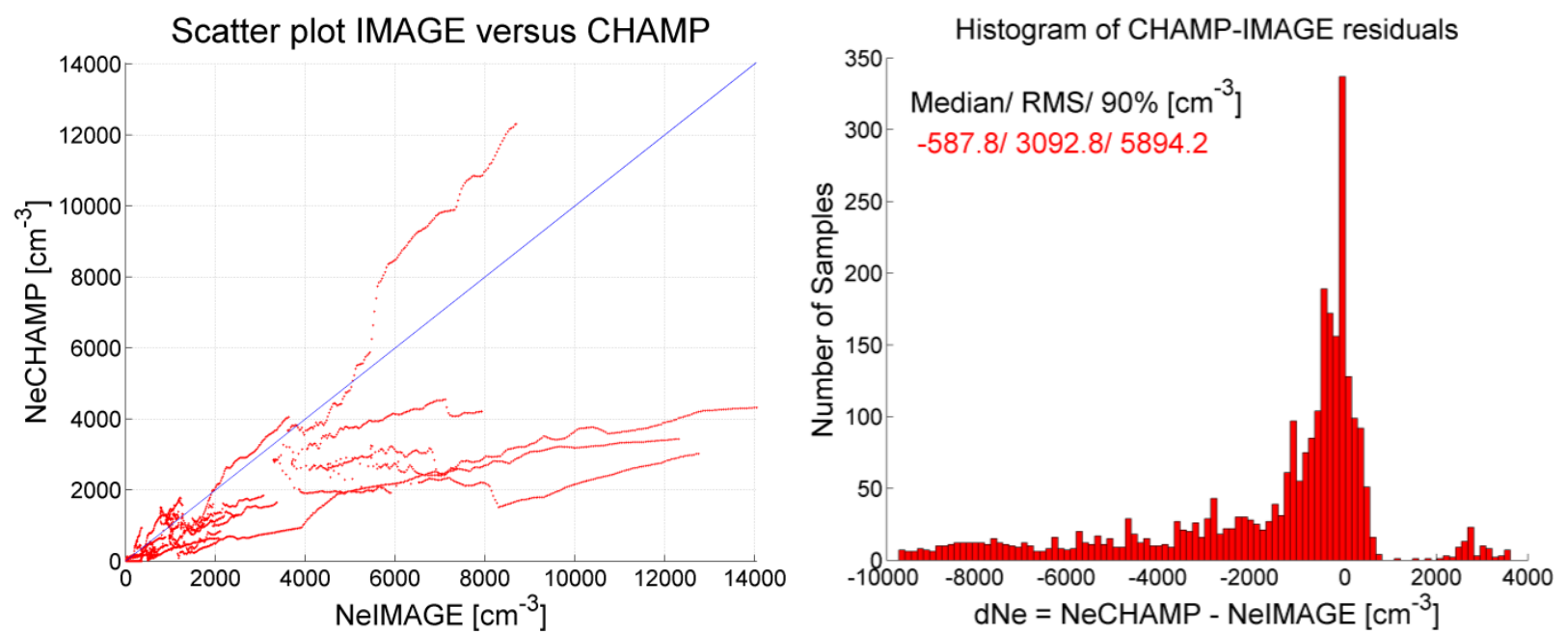

Figure 3: Left: Scatter plot of the IMAGE profiles (horizontal) versus CHAMP (vertical) electron density values. Right: The histogram of the $d N e$ values.
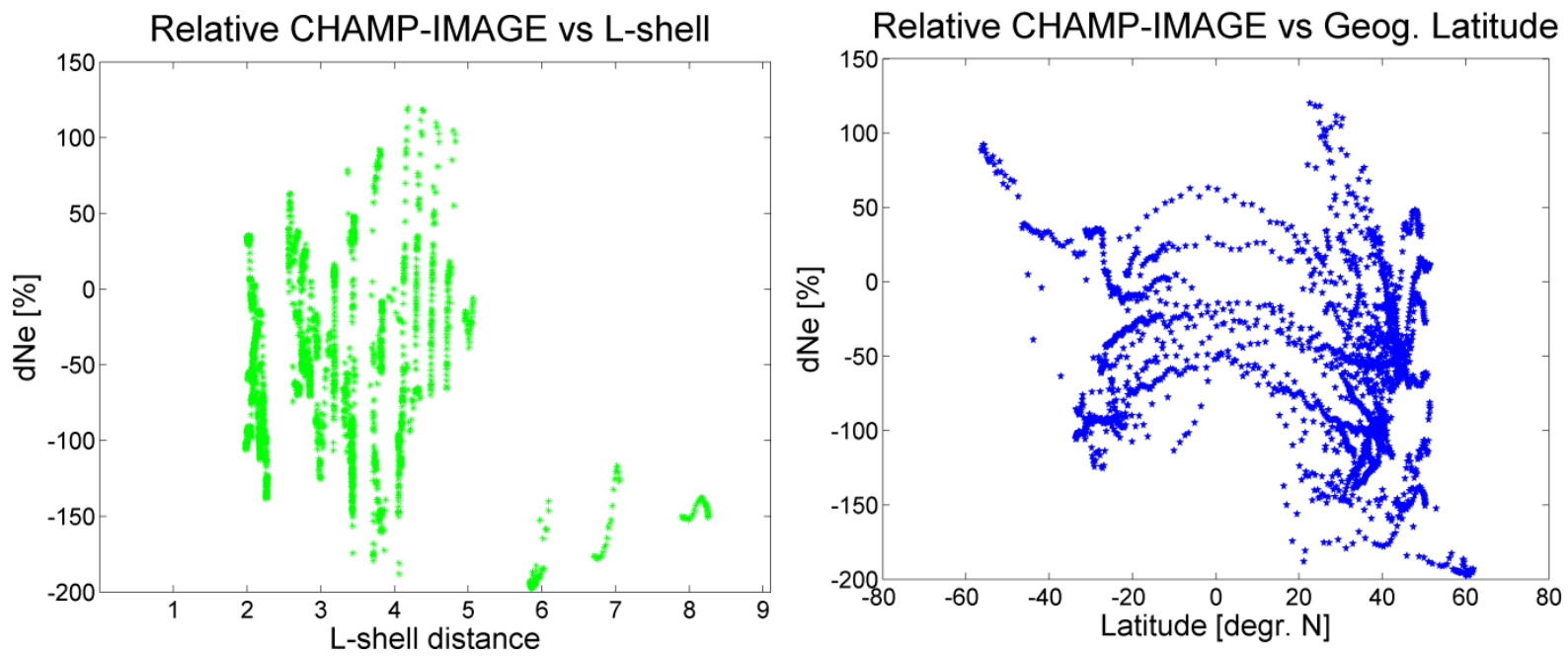

Figure 4: $d N e_{\%}$ values versus L-shell (left) and versus geographic latitude (right)。 

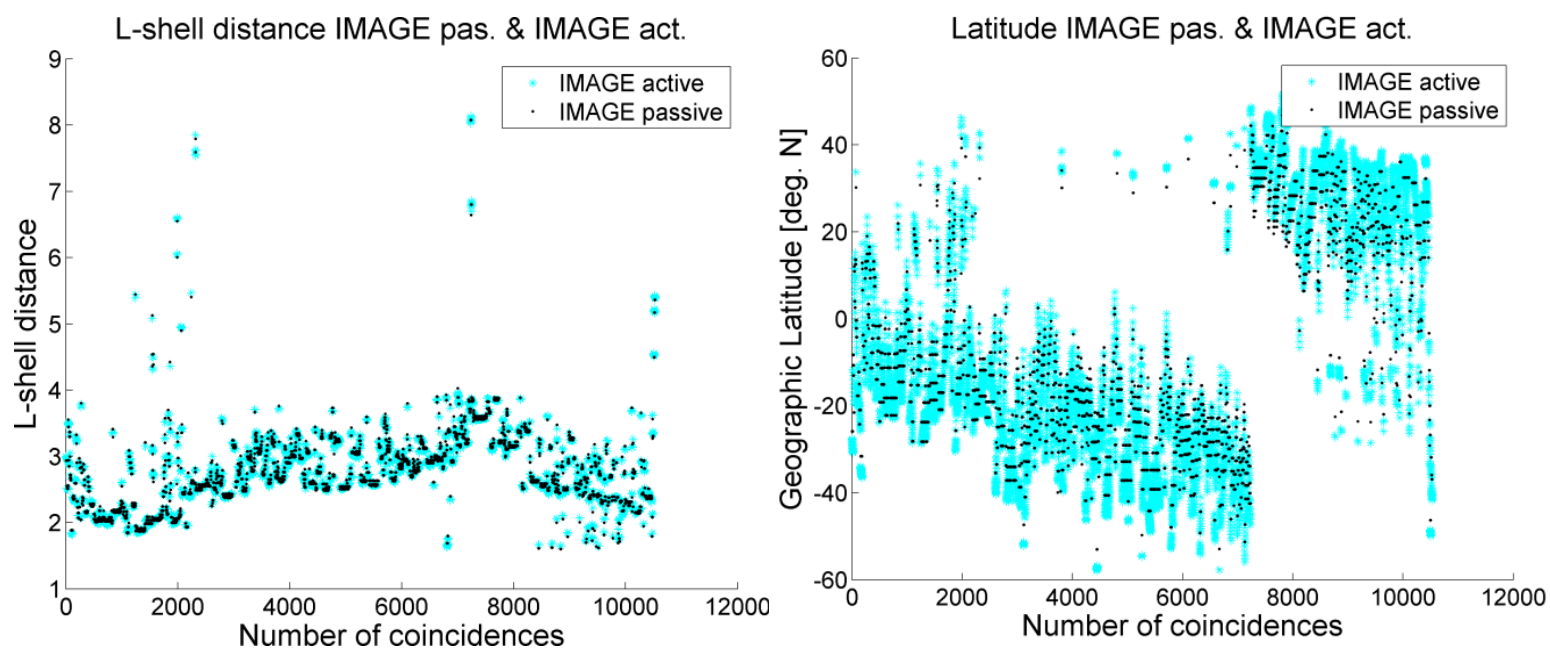

Figure 5: L-shell (left) and geographic latitude (right) of the IMAGE active (cyan stars) and passive (black dots) coincident data.
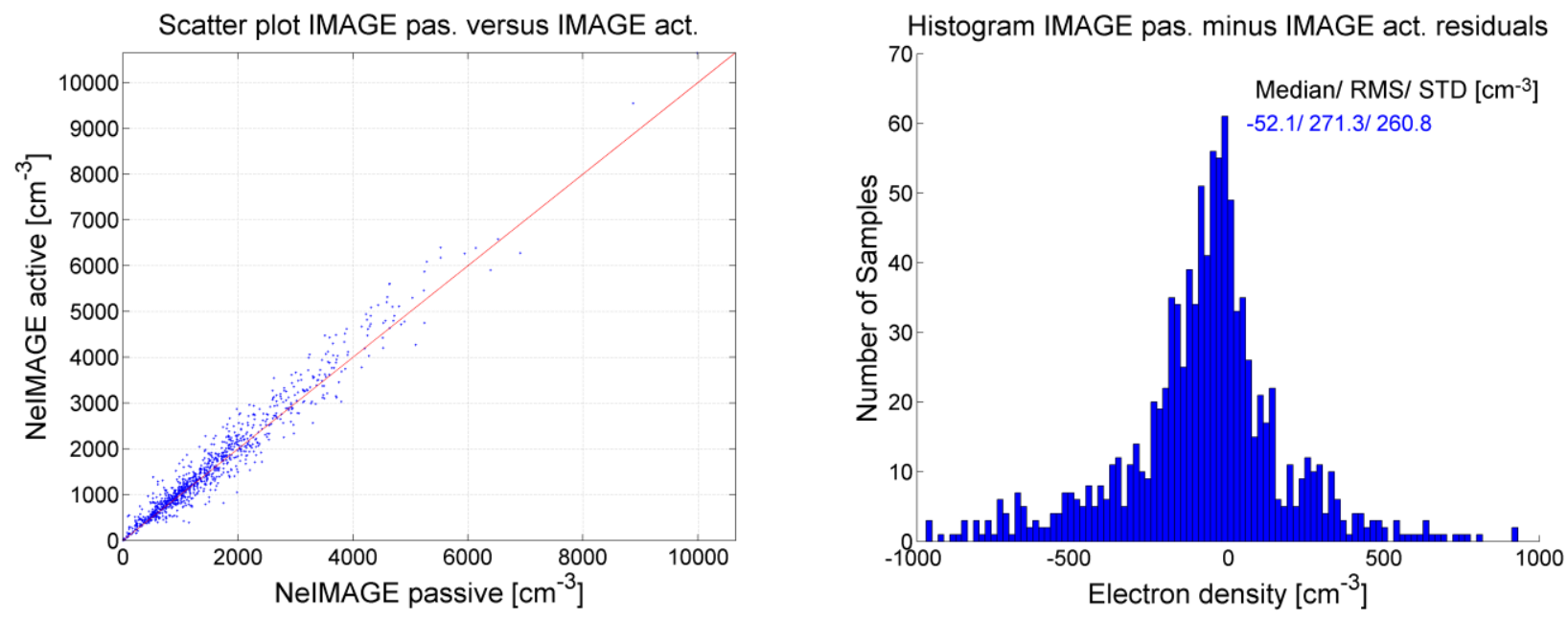

Figure 6: Left: Scatter plot of the IMAGE passive (horizontal) versus averaged profiles (vertical) electron density values. Right: The histogram of the IMAGE passive minus active residuals. 

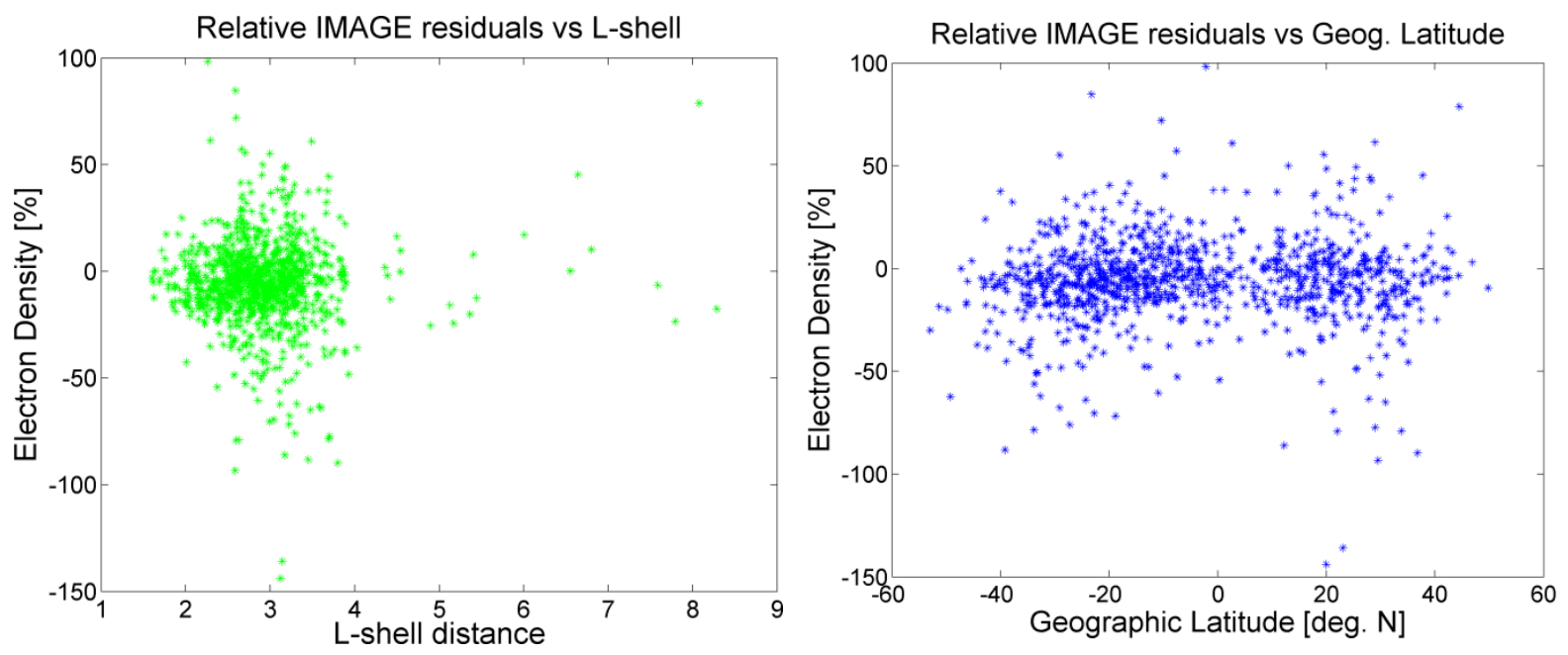

Figure 7: Relative IMAGE residuals versus L-shell (left) and versus geographic latitude (right).
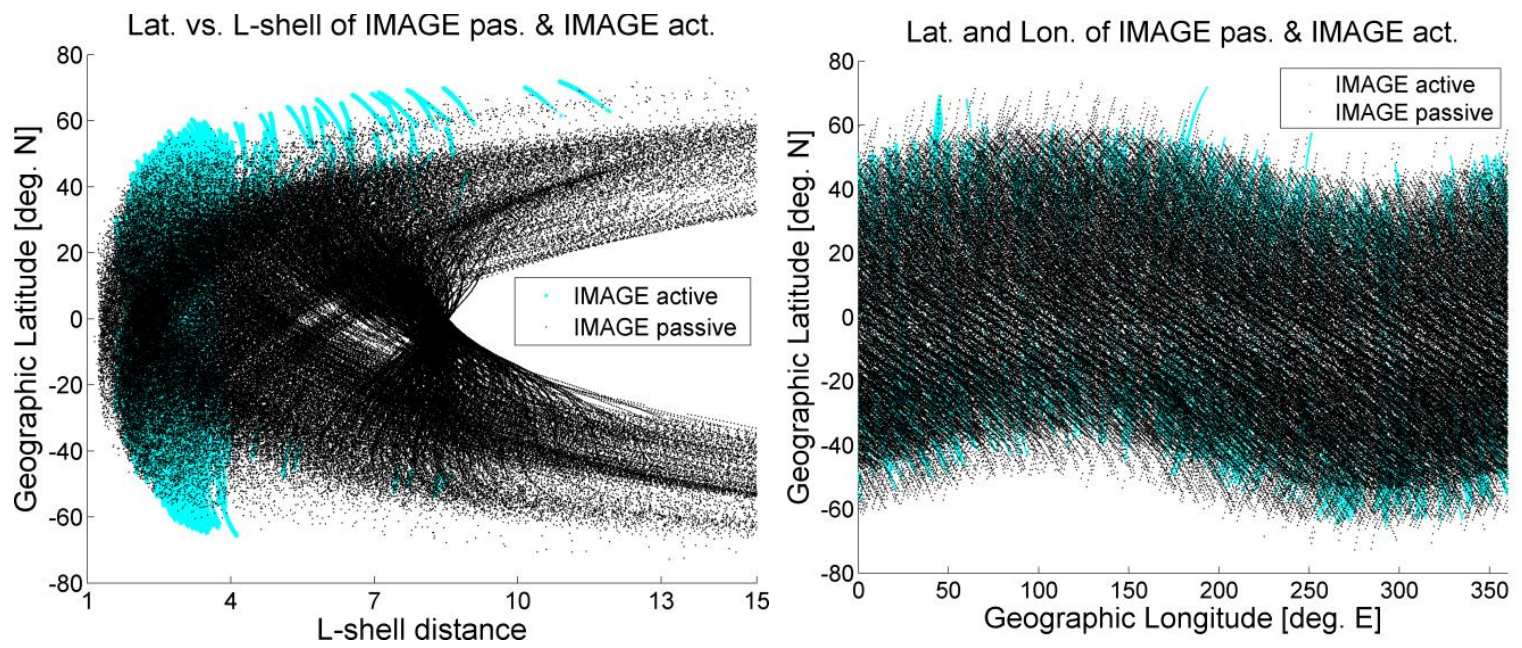

Figure 8: Locations of all available IMAGE active (cyan) and passive (black) observation points for years 2001-2005. Left: Geog. longitude versus latitude, Right: Geog. latitude versus L-shell. 


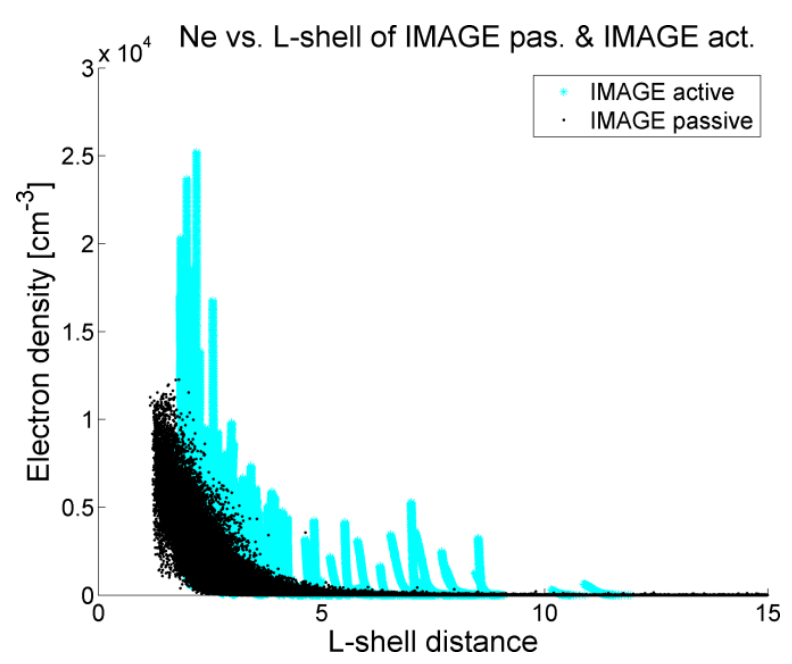

Figure 9: IMAGE electron density (passive - black and active - cyan) versus L-shell for years 20012005.
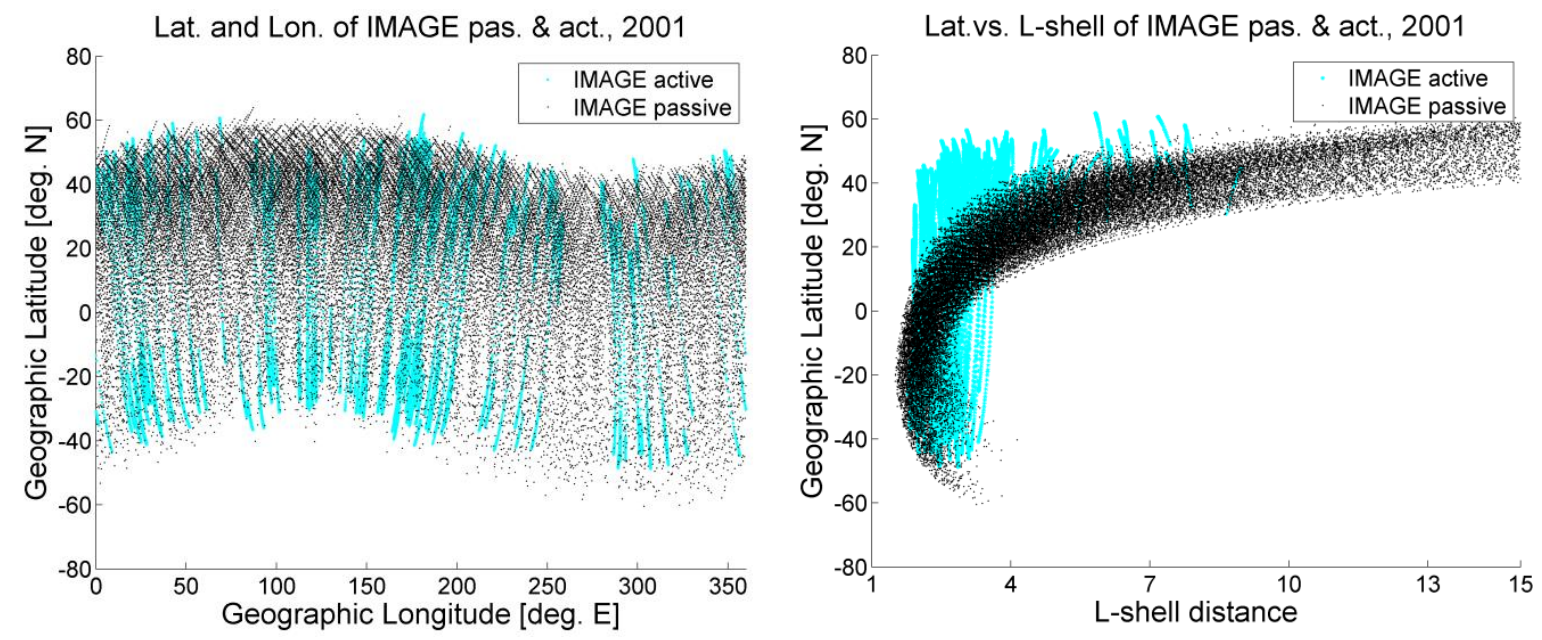

Figure 10: Locations of all available IMAGE active (cyan) and passive (black) observation points for year 2001. Left: Geog. longitude versus latitude. Right: Geog. latitude versus L-shell.

Table 1: The statistics of the $d N e_{\%}$ values binned to L-shell ranging between (from top to bottom): 1 and $3 ; 3$ and $4 ; 4$ and 8.

\begin{tabular}{|l|l|l|l|l|}
\hline & Median & RMS & $\mathbf{9 0 \%}$ & Samples \\
\hline L-shell distance below 3 & -44.93 & 51.31 & 71.84 & 1460 \\
\hline L-shell between 3 and 4 & -61.18 & 62.29 & 82.13 & 882 \\
\hline L-shell distance above 4 & -36.27 & 78.41 & 99.17 & 419 \\
\hline
\end{tabular}

